\title{
Emission measurement and safety assessment for the production process of silicon nanoparticles in a pilot-scale facility
}

\author{
Journal Article \\ Author(s): \\ Wang, Jing; Asbach, Christof; Fissan, Heinz; Hulser, Tim; Kaminski, Heinz; Kuhlbusch, Thomas A.J.; Pui, David Y.H. \\ Publication date: \\ 2012-04 \\ Permanent link: \\ https://doi.org/10.3929/ethz-b-000048210 \\ Rights / license: \\ In Copyright - Non-Commercial Use Permitted \\ Originally published in: \\ Journal of Nanoparticle Research 14(4), https://doi.org/10.1007/s11051-012-0759-y
}




\title{
Emission measurement and safety assessment for the production process of silicon nanoparticles in a pilot- scale facility
}

\author{
Jing Wang • Christof Asbach • Heinz Fissan • \\ Tim Hülser • Heinz Kaminski • \\ Thomas A. J. Kuhlbusch • David Y. H. Pui
}

Received: 17 November 2011 / Accepted: 27 January 2012/Published online: 10 March 2012

(C) Springer Science+Business Media B.V. 2012

\begin{abstract}
Emission into the workplace was measured for the production process of silicon nanoparticles in a pilot-scale facility at the Institute of Energy and Environmental Technology e.V. (IUTA). The silicon nanoparticles were produced in a hot-wall reactor and consisted of primary particles around
\end{abstract}

Special Issue Editors: Candace S.-J. Tsai, Michael

J. Ellenbecker

This article is part of the Topical Collection on

Nanotechnology, Occupational and Environmental Health

J. Wang $(\varangle)$

Institute of Environmental Engineering, ETH Zurich, 8093 Zurich, Switzerland

e-mail: jing.wang@ifu.baug.ethz.ch

J. Wang

Analytical Chemistry, Empa, 8600 Dübendorf,

Switzerland

C. Asbach · H. Fissan - T. Hülser · H. Kaminski .

T. A. J. Kuhlbusch

Institute of Energy and Environmental Technology e.V.

(IUTA), 47229 Duisburg, Germany

H. Fissan · T. A. J. Kuhlbusch

Center for Nanointegration Duisburg-Essen (CeNIDE), 47057 Duisburg, Germany

D. Y. H. Pui

Particle Technology Laboratory, University of Minnesota, Minneapolis 55414, USA
$60 \mathrm{~nm}$ in diameter. We employed real-time aerosol instruments to measure particle number and lungdeposited surface area concentrations and size distribution; airborne particles were also collected for off-line electron microscopic analysis. Emission of silicon nanoparticles was not detected during the processes of synthesis, collection, and bagging. This was attributed to the completely closed production system and other safety measures against particle release which will be discussed briefly. Emission of silicon nanoparticles significantly above the detection limit was only observed during the cleaning process when the production system was open and manually cleaned. The majority of the detected particles was in the size range of $100-400 \mathrm{~nm}$ and were silicon nanoparticle agglomerates first deposited in the tubing then re-suspended during the cleaning process. Appropriate personal protection equipment is recommended for safety protection of the workers during cleaning.

Keywords Silicon nanoparticles · Emission measurement $\cdot$ Workplace safety $\cdot$ Nanoparticle agglomerates

\section{Introduction}

Sustainability issues, and environmental health and safety (EHS) aspects of nanomaterials are becoming a focus with the development of nanotechnology (Wang et al. 2011). The National Institute of Occupational 
Safety and Health (NIOSH, US) has designated EHS impact of nanomaterials as one of the focuses of the strategic plan (NIOSH 2009). NIOSH published the recommended exposure limits (RELs) for fine and ultrafine titanium dioxide (NIOSH 2011) and draft RELs for carbon nanotubes and nanofibers (NIOSH 2010).

Data of occupational exposure to different types of nanomaterials play an important role in determining the health effect on workers. A number of studies have been dedicated to emission and workers' exposure during production or handling of carbon nanofibers and nanotubes (Mazzuckelli et al. 2007; Han et al. 2008; Tsai S et al. 2009a; Wang and Pui 2011). Kuhlbusch et al. (2004, 2006) studied particle emission in carbon black production. Demou et al. (2008) and Seipenbusch et al. (2008) investigated temporal evolution of nanoparticles in workplace exposure; Walser et al. (2012) studied both temporal and spatial evolution of released nanoparticles under accident situations. Tsai C-J et al. (2009) measured the dustiness when nanoparticles are handled in a rotating drum. Tsai S et al. (2009b) measured exposures of nanoalumina and nanosilver during manual handling in fume hoods. Reports of workplace exposures suggest that engineered nanomaterials are released at high mass and/or number concentrations only under unusual circumstances (Han et al. 2008; Kuhlbusch et al. 2004; Kuhlbusch and Fissan 2006; Maynard et al. 2004). Brouwer (2010) and Kuhlbusch et al. (2011) reviewed recent exposure studies at nanotechnology workplaces. With the development of new nanotechnologies and nanomaterials, there still exists the need for study of exposure levels in occupational and environmental settings. Only a holistic approach assessing potential exposure and hazard during the entire life cycle will lead to general acceptance of "nano-products" and hence guarantee sustainable development of the nanotechnology industry.

\section{Production cycle of silicon nanoparticles in the pilot scale plant}

We performed emission measurement at a pilot-scale nanoparticle production plant at the Institute of Energy and Environmental Technology e.V. (IUTA), Duisburg, Germany. The facility is designed to produce nanoparticles in the kilograms/day range.

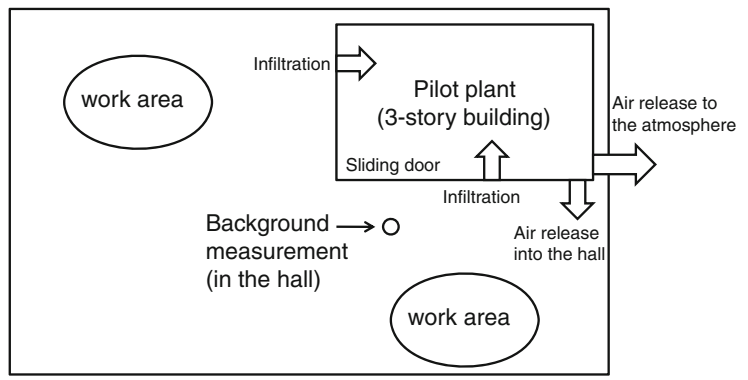

Fig. 1 A schematic for the pilot-scale production plant

The pilot plant is a three-story building within a large hall, and it is separated from the rest of the hall by acrylic sheets at the upper levels and sliding doors at the ground level, though air exchange in and out of the plant is still possible through the gaps around the sliding door. The air flow between the floors is unhindered due to the use of metal grids as a floor. The ventilation system located at the top of the plant draws air out of the plant and can either release the air into the hall during standby or release it to the atmosphere outside of the hall during production. Due to the gaps on the ground level, the ventilation system generates a continuous flow of fresh air that crosses the pilot plant and, furthermore, produces a slightly negative pressure during operation within the facility. Outside the pilot plant, there exist other working areas in the hall. A schematic of the plant is shown in Fig. 1.

Our measurement covered the whole production cycle of silicon nanoparticles, including generation from the reactor, collection by filters, bagging,

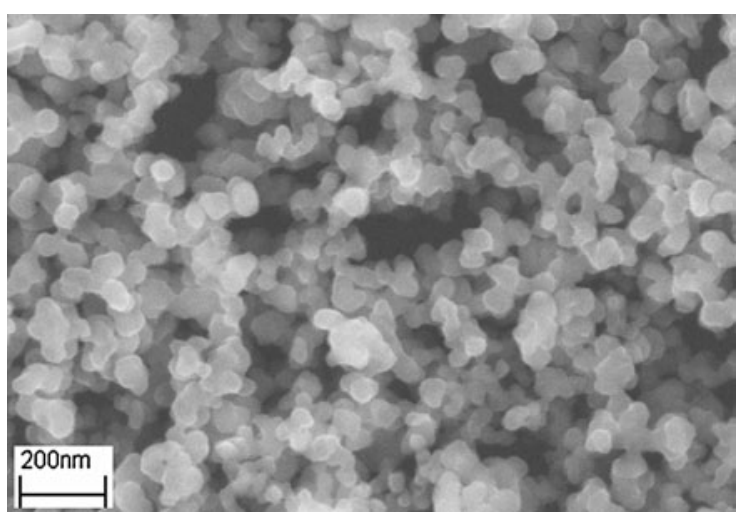

Fig. 2 A scanning microscopic image of the Si nanoparticles produced by the hot-wall reactor 


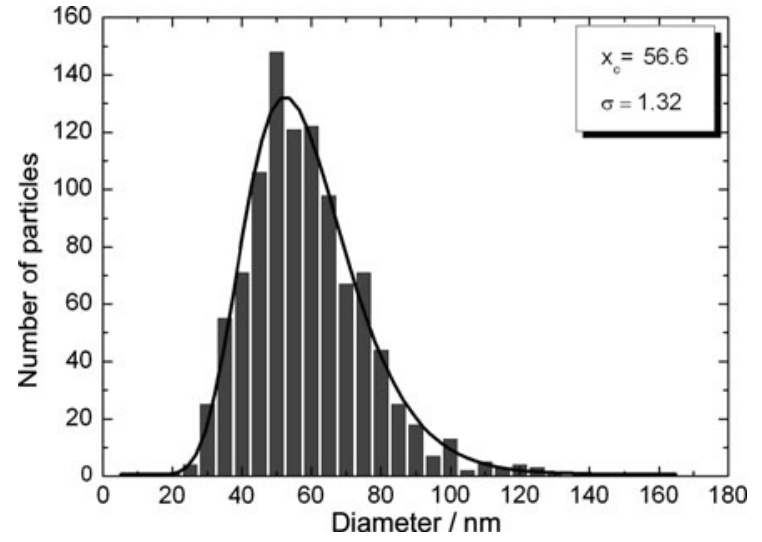

Fig. 3 The size distribution of the primary $\mathrm{Si}$ particles produced by the hot-wall reactor

packaging, and cleaning of the system. Silicon nanoparticles were produced in a hot-wall reactor, in which the precursor gas was thermally decomposed to form the nanoparticles. Figure 2 shows an example of the scanning electron microscopic (SEM) images of the Si nanoparticles. Image analyses of the SEM data for about 1,000 primary particles were used to determine the size distribution shown in Fig. 3, which had a peak at $57 \mathrm{~nm}$ and a geometric standard distribution of 1.32. The agglomerates formed by the primary particles were in the range of several hundred nanometers. The generated particles were covered by a naturally grown oxide layer on the surface, but the core part was pure silicon (Hülser et al. 2010). The reactor was connected through tubing to the filter housing where the particles were collected on filters. To enable continuous operation a double-filter system was installed. The particles were filtered alternately by one of the filter cartridges, while reverse pulsing was applied to the other one to blow the particles off the filter, which then dropped into a plastic bag underneath. The plastic bag was then sealed, removed from the system, and made ready for shipping. The bagging process is completely sealed so that a release of the particles can only be expected in case of an accident. The cleaning process involved purging the tubing system with pressurized air, opening the tubing system and manual cleaning. The double filter system also enables to separate particles that are generated in the first stages of production from those that are synthesized later under the desired stable operating conditions (Hülser et al. 2010).

\section{Emission measurement}

Instrumentation

A suite of aerosol instruments were used for the measurement of nanoparticle emission into air, including the Fast Mobility Particle Sizer (FMPS, TSI model 3091, particle size range 5.6-560 nm, $1 \mathrm{~s}$ time resolution), Nanoparticle Surface Area Monitor (NSAM, TSI model 3550, particle size range $<1 \mu \mathrm{m}$, $1 \mathrm{~s}$ time resolution, alveolar deposition mode), Ultrafine Water-based Condensation Particle Counter (UWCPC, TSI model 3786, particle size range $>2.5 \mathrm{~nm}, 1 \mathrm{~s}$ time resolution), Handheld Condensation Particle Counter (CPC, TSI model 3007, particle size range $>10 \mathrm{~nm}, 1 \mathrm{~s}$ time resolution), and Scanning Mobility Particle Sizer (SMPS, TSI model 3936 with long DMA, $0.3 \mathrm{~L} / \mathrm{min}$ aerosol flow rate, $3 \mathrm{~L} / \mathrm{min}$ sheath flow rate, size range $15-750 \mathrm{~nm}, 5 \mathrm{~min}$ time resolution). A second set of the instruments were deployed outside of the enclosure of the pilot plant to monitor the particle level at the background; the location for the background measurement is indicated in Fig. 1. This approach was pursued to enable differentiation of particles released by the production processes from those generated by other sources in the background. The comparability of the two sets of instruments was verified by performing side by side measurements prior to actual field measurements and any differences were taken into account in the data interpretation. The total particle number concentration from the FMPS used for the background was higher than the one used in the pilot plant; the offset was about $1,500 \# / \mathrm{cm}^{3}$. The lung-deposited surface area from the NSAM used in the plant was higher than the one used for the background by about $7 \mathrm{~mm}^{2} / \mathrm{cm}^{3}$.

Conductive silicone tubing with $0.48-\mathrm{cm}$ ID and about 1-m length was used for sampling purpose. The conductive tubing effectively reduced electrostatic loss so that diffusion loss was the only major mechanism for nanoparticles during transportation. The diffusion loss computed by the Gormley and Kennedy (1949) formula was less than $1.5 \%$ for particles larger than $30 \mathrm{~nm}$ for $1-\mathrm{m}$ tubing and 2.5-L/min flow rate. In addition, the readings of FMPS and NSAM were almost identical before and after attaching the tubing to the instrument inlets. Thus, we did not implement additional corrections on the results 
due to the sampling tubing. We collected airborne particle samples using the Nanometer Aerosol Sampler (NAS, TSI 3089). The particles were electrostatically deposited on a silicon chip. Subsequent SEM and energy dispersive X-ray (EDX) analyses were performed.

\section{Overnight measurement}

We performed overnight measurements using the two sets of FMPS and NSAM, which provided the airborne particle levels when there was no activity in the plant or in the hall. The overnight measurement results of the two FMPS units are shown in Fig. 4. Measurements from the two FMPS units were consistent, despite the offset of about $1,500 \# / \mathrm{cm}^{3}$. The total airborne particle concentration started to decrease around 18:00 after the workers left and reached the

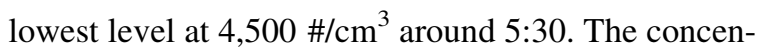
tration then started to increase due to the worker activities in the morning. The overnight measurements of the two NSAM units (not shown) were similar to those of the FMPS units, also demonstrating good consistency. The particle size distributions obtained during the overnight measurement in the pilot plant and in the hall are shown in Fig. 5a, b, respectively. It can be seen that the size distributions in the plant and in the hall at the same time were similar. The main peak of the size distributions was about 40-60 nm, and the peak location did not change much overnight. At 10:00, the particle concentration was noticeably higher due to worker activities.

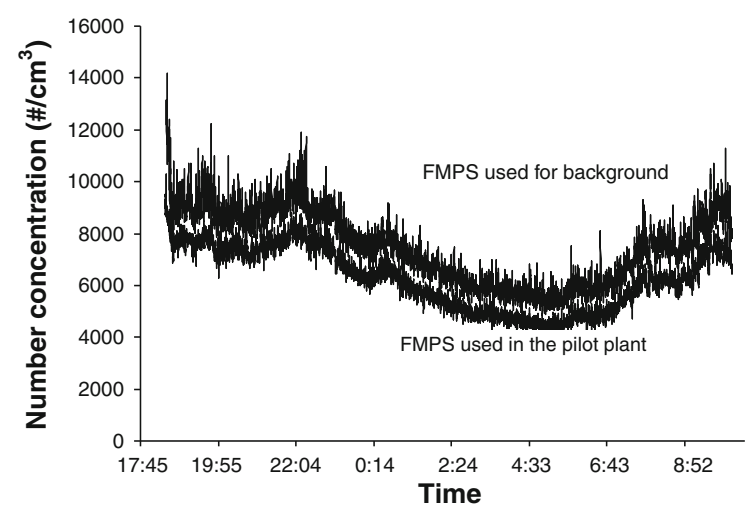

Fig. 4 Overnight measurement of the total particle number concentration from the two FMPS units
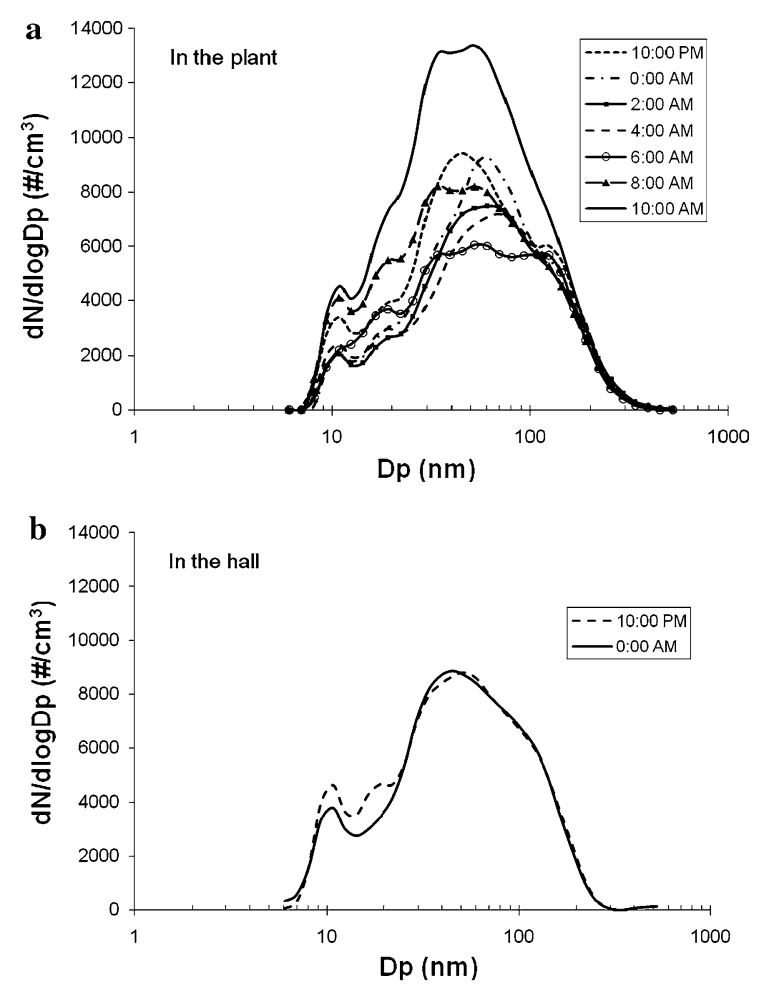

Fig. 5 a The particle size distribution measured overnight in the pilot plant. b The particle size distribution measured overnight in the hall outside the plant

Measurement during the production process

We placed the instruments in the immediate vicinity of the hot-wall reactor and measured the possible emission during production of Si nanoparticles. The reactor was located on the third floor in the plant; the carrier gas from the reactor flowed through tubing to the filter housing on the second floor where the particles were collected on filters. The air exchange between the floors is unrestricted due to the use of metal grids for floors. The production was carried out in the completely closed system. Figure 6 shows the total particle number concentration (derived from FMPS measurements) and the NSAM reading for lung-deposited surface area concentration during the production process. Results both in the plant and background are shown. The offsets between the instruments contributed to discrepancies between the curves. It can be seen that the concentrations in the plant and background had the same trend. There was no evidence for particle concentration increase in the 
plant due to nanoparticle production. Some concentration fluctuations occurred in the background, presumably due to worker activities outside the production plant. We also deployed the UWCPC (TSI model 3786) near the hot-wall reactor, which gave the total particle number concentration around $1.2 \times 10^{4} \mathrm{\#} / \mathrm{cm}^{3}$, close to the result given by the FMPS. The particle size distributions measured in the plant and background during production are shown in Fig. 7a, b, respectively. We observed relatively high concentrations in the background at 12:00, 13:00, and 14:50, which were presumably due to worker activities in the hall including mechanical machining. The distributions in the plant were similar to those in the background when the background concentration was low. The results indicated no emission from the production process. We measured the total particle number concentrations at different locations in the production plant with the Handheld CPC (TSI model 3007). The concentration near the hot-wall reactor

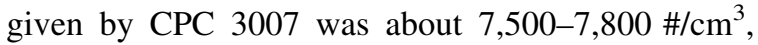
lower than the reading of CPC 3786 . The difference was attributed to the different size detection limits: $2.5 \mathrm{~nm}$ for CPC 3786 and $10 \mathrm{~nm}$ for CPC 3007. We measured the concentrations at a number of locations on each floor, and they were all between 7,200 and $8,200 \mathrm{\#} / \mathrm{cm}^{3}$. Thus, no emission was detected throughout the entire plant during production since the flow was from the ground floor to the third floor, where the measurement equipment was located. We concluded that the closed system for the production very effectively contained the produced nanoparticles.

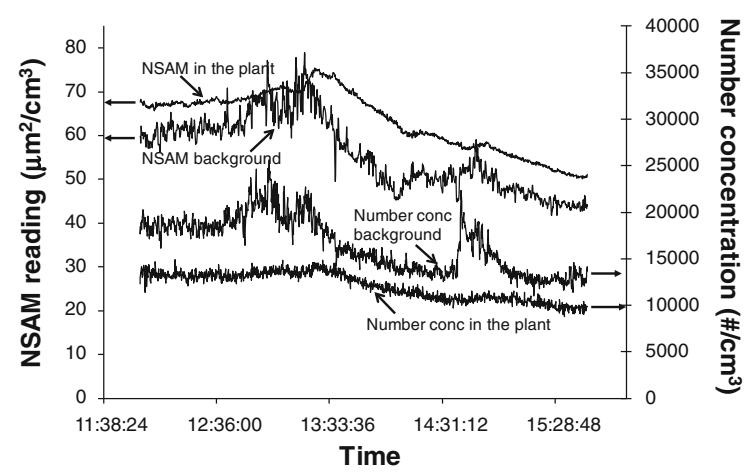

Fig. 6 The total particle number concentration and the NSAM reading for lung-deposited surface area concentration during the production process. Both the measurements in the plant and at the background are shown
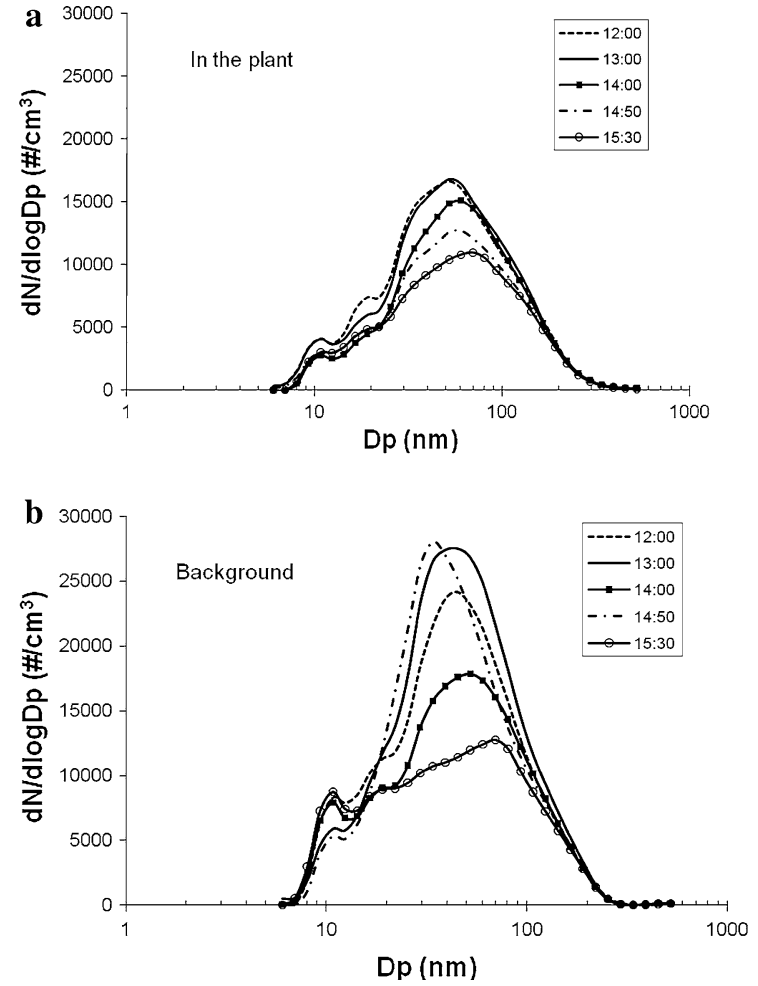

Fig. 7 a The particle size distribution measured near the hotwall reactor during Si particle production. b The particle size distribution measured in the background during $\mathrm{Si}$ particle production

We placed the NAS sampler close to the pump which controlled the pressure in the hot-wall reactor. The sampling flow rate was about $2 \mathrm{~L} / \mathrm{min}$ and the sampling time was about 2.5 hours Subsequent SEM found sub-micron particles of different morphologies and the EDX analysis revealed that these particles were not Si particles from the hot-wall reactor. The result confirmed that $\mathrm{Si}$ particles were not emitted during the production process.

Measurement during the process of bagging and packaging

After collection of the produced Si nanoparticles on the baghouse filters, the process of bagging and packaging started. The filter housing on the second floor was connected by a vertical pipe which extended to the height of about $1 \mathrm{~m}$ above the ground. The vertical pipe consisted of two stages of valves and a lock (Fig. 8). A plastic bag was mounted at the end of 


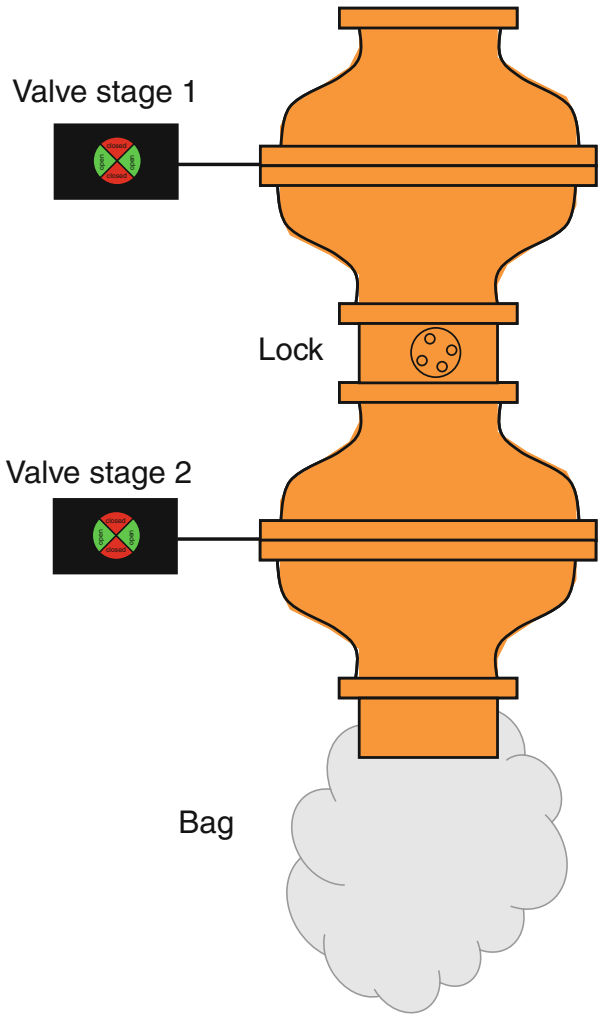

Fig. 8 An illustration of the vertical pipe connecting the filter housing and the receiving bag

the pipe to receive the nanoparticles. To start the bagging process, the lock was first opened by compressive air. Then reverse pulsing of the filter housing was carried out firstly with valve 1 open and valve 2 closed. Then, valve 1 was closed and valve 2 was open, and the particles dropped into the bag. The second-stage reverse pulsing was performed with both valve 1 and 2 open. This procedure of reverse pulsing was repeated several times to transport the maximum amount of particles into the bag. The bag was mounted on the pipe with double clamps. Once the reverse pulsing was finished, the bag was cut off the pipe with scissors. Finally the bag was sealed with a hot bar sealer to finish the packaging process.

We placed the FMPS and NSAM close to the receiving bag on the ground floor. We positioned the inlets of the sampling tubes approximately $20 \mathrm{~cm}$ above the section where the bag was clamped to the pipe. Continuous measurement by the FMPS and NSAM during the bagging and packaging process showed no obvious increase of the particle concentration, excepted for the thermal seal process when the

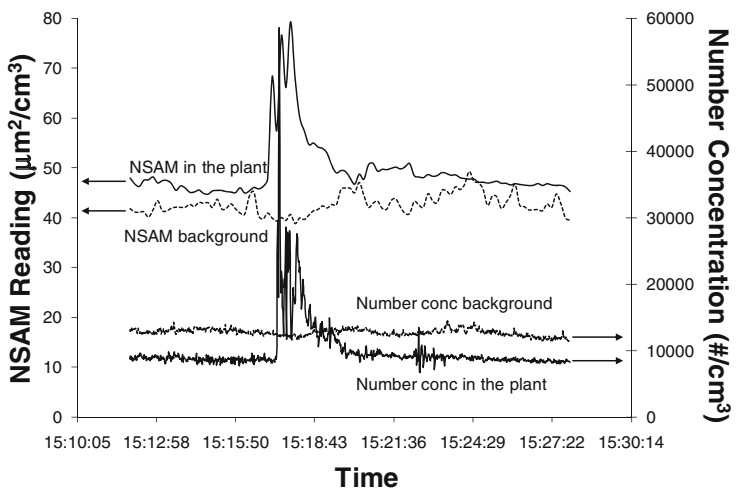

Fig. 9 The total particle number concentration and the NSAM reading for lung-deposited surface area concentration in the time period around the thermal seal process

bag was sealed by the hot bar sealer. The total particle number concentration and the NSAM reading in the selected time period around the thermal seal process are shown in Fig. 9. The jump of the particle concentration starting around $15: 16$ was due to the thermal seal process, in which the neck of the bag was placed between a pair of hot metal bars and pressed to be sealed. The total number concentration jumped from 8,500 to $58,500 \mathrm{\#} / \mathrm{cm}^{3}$; the NSAM reading jumped from 42 to $79 \mu \mathrm{m}^{2} / \mathrm{cm}^{3}$. The particle size distributions before (15:15:00), during (15:17:40 and $15: 18: 20)$, and after $(15: 20: 00)$ the thermal seal process are shown in Fig. 10. The particle concentration in the size range of $20-60 \mathrm{~nm}$ increased significantly during the thermal seal process. We believe that these particles are organic particles formed by condensation of the evaporated plastic material from

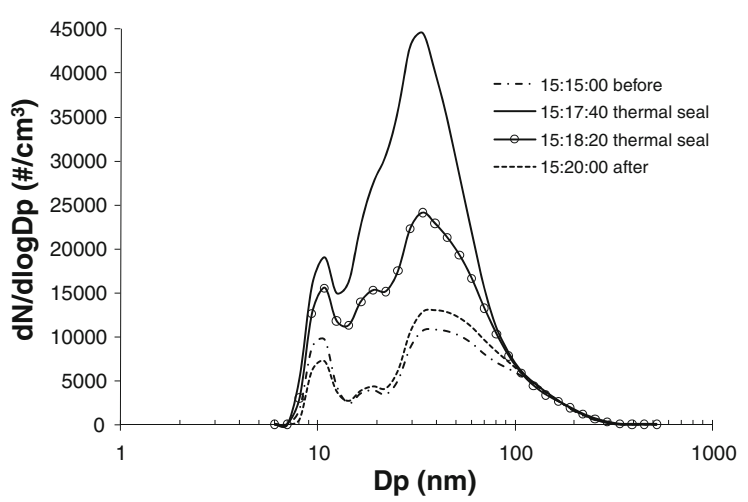

Fig. 10 The particle size distribution measured in the time period around the thermal seal process. The two curves for $15: 17: 40$ and 15:18:20 correspond to the thermal seal process 
the bag at high temperatures, because such particle concentration jump occurred only when the thermal seal was performed.

We found that the safety measures were adequate to prevent emission of the produced nanoparticles during bagging and packaging process. The technicians who performed removal and thermal seal of the bag wore respirators during the operation, which were highly efficient against particles in the nanometer range.

Measurement during the cleaning of the system

The synthesis system, including the reactor, the tubing system, and the filters, needs to be cleaned when the production conditions change to avoid contamination of the products. We measured possible particle emission during the cleaning after production of $\mathrm{Si}$ nanoparticles. The cleaning procedure consisted of two major steps. In the first step, a vacuum pump was connected to the synthesis system and the pressure was pumped down to 50 mbar. Valves in the system were open and shut with a periodicity of a few seconds to inject $\mathrm{N}_{2}$ into the system in order to remove residual particle on the walls of the reactor and tubing. The system pressure increased to about $300 \mathrm{mbar}$ due to $\mathrm{N}_{2}$ injection. A filter was installed between the pump and the synthesis system to collect the residual particles. The vacuum pump was switched off after about 25 min of cleaning. In the second step, different tubing sections were disconnected and manually vacuumcleaned. All the technicians and researcher put on respirators before opening the system. Substantial particle deposition on the inside walls of tubing was visible. The technicians then switched on the vacuum pump and used the pipe connected to it to draw the particles off the tubing wall. The tubing was knocked on by a wrench to dislodge particles from the wall. After cleaning of the tubing, the vacuum pump and its pipe were wiped with isopropyl alcohol-saturated wipes.

We placed the FMPS and NSAM underneath the tubing system on the second floor during the cleaning process. The total particle number concentration from

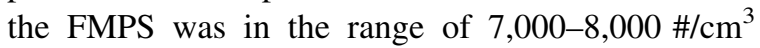
during 10:50 to 11:05, before cleaning started. It increased gradually to $8,000-9,000 \# / \mathrm{cm}^{3}$ during 11:05 to 11:30, when cleaning with the $\mathrm{N}_{2}$ injection was performed. Correspondingly, the NSAM reading increased approximately from $38 \mu^{2} / \mathrm{cm}^{3}$ at $10: 50$ to
$49 \mu \mathrm{m}^{2} / \mathrm{cm}^{3}$ at 11:30. After opening of the system and start of the manual cleaning at 11:38, the particle concentrations continued to increase gradually. At 12:00, the total particle number concentration was approximately $10,000 \# / \mathrm{cm}^{3}$ and the NSAM reading was approximately $60 \mu \mathrm{m}^{2} / \mathrm{cm}^{3}$. The concentrations then became steady except when the tubing was knocked on by a wrench.

We used the FMPS and NSAM to measure emission during manual cleaning of the tubing sections of the production system. We placed the inlets of the sampling tubes at the opening of the tubing to capture the emitted particles. We observed spikes of the particle concentrations when the system tubing was knocked on with a wrench, as shown in Fig. 11. The total number concentration reached $17,000 \mathrm{\#} / \mathrm{cm}^{3}$, more than twice of that before cleaning; the NSAM reading reached $174 \mu \mathrm{m}^{2} / \mathrm{cm}^{3}$, more than

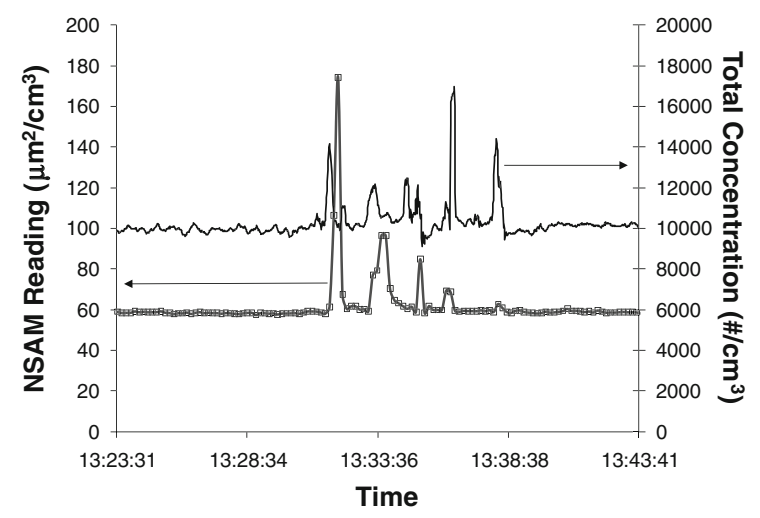

Fig. 11 The total particle number concentration and the NSAM reading for lung-deposited surface area concentration during the manual cleaning process. The spikes were observed when the tubing of the production system was knocked on by a wrench

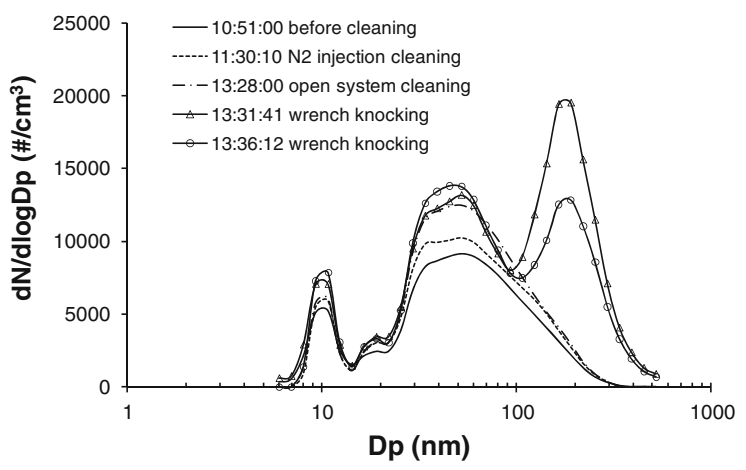

Fig. 12 The particle size distributions at different stages of the cleaning process 
four times of that before cleaning. The particle size distributions at different stages of the cleaning process are shown in Fig. 12. It can be seen that the particle concentration increased after the cleaning started, and it increased further after the tubing system was open. The shape of the distributions did not change significantly except when the tubing system was knocked on by a wrench. In the case of wrench knocking, a new mode for particles between 100 and $400 \mathrm{~nm}$ appeared in the size distribution. It was a clear indication that the particles were dislodged off the wall and became airborne, and these particles were mostly in agglomerated form and thus in the size range of 100-400 nm. It should be noted that the spikes of the particle concentrations were measured at the opening of the tubing sections of the production system, therefore they might be higher than the concentrations in the breathing zone. Nevertheless, the measurement demonstrated the emission source of particles during manual cleaning, and showed that the particles were in the inhalable size range. The instruments deployed in our study were sensitive to particles in the size range below approximately $1 \mu \mathrm{m}$. It is possible that larger and inhalable particles were released during the cleaning process but were not detected by our instruments. Tsai et al. (2011) showed that the mass median aerodynamic diameter was in the range of 4.61-6.15 $\mu \mathrm{m}$ in their measurement at three different nanopowder workplaces. Our results indicated that potential exposure risks existed during the cleaning process. To obtain quantitative exposure results, further personal exposure studies are needed.

\section{Conclusion}

Our results showed that the particle concentration in the production facility were comparable with that in the background for the majority of the processes we measured. Nanoparticle release from the manufacturing process can be minimized with proper procedures and the closed system is very effective in containing produced nanoparticles, assuring safe production. However, protection for workers is still advisable during special processes, such as cleaning and packaging, when hazardous substances or substances with unknown hazard potential are handled. The protection strategies include wearing respirators, face masks, and usage of effective ventilation systems (Walser et al.
2012). Furthermore there is a strong need to study potential release of and exposure to nanomaterials during further (industrial) processing steps, use by consumers and recycling.

Acknowledgments The work was partially supported by the National Institute of Environmental Health Sciences Grant \# 1RC2ES018741-01 (Sub-Grant 100029-D) on "Hazard Assessment and Risk Estimation of Inhaled Nanomaterials Exposure" and by the NSF grant (Award ID: 0646236) on "Experimental and Numerical Simulation of the Fate of Airborne Nanoparticles from a Leak in a Manufacturing Process to Assess Worker Exposure". The emission measurements were performed during the production cycle of silicon nanoparticles at IUTA e.V. The authors thank the production team: Mathias Spree, Stefan Berthold, Christof Jurczyk and Frank Krumpolt for collaboration.

\section{References}

Brouwer D (2010) Exposure to manufactured nanoparticles in different workplaces. Toxicology 269:120-127

Demou E, Peter P, Hellweg S (2008) Exposure to manufactured nanostructured particles in an industrial pilot plant. Ann Occup Hyg 52(8):695-706

Gormley PG, Kennedy M (1949) Diffusion from a stream flowing through a cylindrical tube. Proc Roy Irish Acad 52:163-169

Han JH, Lee EJ, Lee JH, So KP, Lee YH, Bae GN, Lee SB, Ji JH, Cho MH, Yu IJ (2008) Monitoring multiwalled carbon nanotube exposure in carbon nanotube research facility. Inhalation Toxicol 20:741-749

Hülser TP, Schnurre SM, Wiggers H, Schulz C (2010) Gasphase synthesis of highly specific nanoparticles on the pilot-plant scale. In: Proceedings of world congress on particle technology (WCPT6)

Kuhlbusch TA, Fissan H (2006) Particle characteristics in the reactor and pelletizing areas of carbon black production. J Occup Environ Hyg 3:558-567

Kuhlbusch TAJ, Neumann S, Fissan H (2004) Number size distribution, mass concentration, and particle composition of PM1, PM2.5 and PM10 in bagging areas of carbon black production. J Occup Environ Hyg 1:660-671

Kuhlbusch TAJ, Asbach C, Fissan H, Göhler D, Stintz M (2011) Nanoparticle exposure at nanotechnology workplaces: a review. Part Fibre Toxicol 8:22

Maynard AD, Baron PA, Foley M, Shvedova AA, Kisin ER, Castranova V (2004) Exposure to carbon nanotube material: aerosol release during the handling of unrefined singlewalled carbon nanotube material. J Toxicol Environ Health Part A 67:87-107

Mazzuckelli JF, Methner MM, Birch ME, Evans DE, Ku B-K, Crouch K, Hoover MD (2007) Case study. Identification and characterization of potential sources of worker exposure to carbon nanofibers during polymer composite laboratory operations. J Occup Environ Hyg 4:125-130

NIOSH (2009) Strategic plan for NIOSH Nanotechnology Research and Guidance: filling the knowledge gaps, DHHS (NIOSH) Publication No. 2010-105 
NIOSH (2010) Current intelligence bulletin: occupational exposure to carbon nanotubes and nanofibers, November 2010 draft

NIOSH (2011) Current intelligence bulletin 63: occupational exposure to titanium dioxide, DHHS (NIOSH) Publication No. 2011-160

Seipenbusch M, Binder A, Kasper G (2008) Temporal evaluation of nanoparticle aerosols in workplace exposure. Ann Occup Hyg 52:707-716

Tsai C-J, Wu C-H, Leu M-L, Chen S-C, Huang C-Y, Tsai P-J, Ko F-H (2009) Dustiness test of nanopowders using a standard rotating drum with a modified sampling train. J Nanopart Res 11:121-131

Tsai S, Hofmann M, Kong J, Hallock M, Ada E, Ellenbecker M (2009a) Nanoparticle release during synthesis of carbon nanotube and the application of filtration. Environ Sci Technol 43(15):6017-6023

Tsai S, Ada E, Isaacs J, Ellenbecker M (2009b) Airborne nanoparticle exposures associated with the manual handling of nanoalumina and nanosilver in fume hoods. J Nanopart Res 11:147

Tsai C-J, Huang C-Y, Chen S-C, Ho C-E, Huang C-H, Chen C-W, Chang C-P, Tsai P-J, Ellenbecker MJ (2011) Exposure assessment of nano-sized and respirable particles at different workplaces. J Nanopart Res 13(9):121-131

Walser T, Hellweg S, Juraske R, Luechinger NA, Wang J, Fierz M (2012) Exposure to engineered nanoparticles: model and measurements for accident situations in laboratories. Sci Total Environ. doi:10.1016/j.scitotenv.2012.01.038

Wang J, Pui DYH (2011) Characterization, exposure measurement and control for nanoscale particles in workplaces and on the road. J Phys Conf Ser 304:012008. doi:10.1088/ 1742-6596/304/1/012008

Wang J, Asbach C, Fissan H, Hülser T, Kuhlbusch TAJ, Thompson D, Pui DYH (2011) How can nanobiotechnology oversight advance science and industry: examples from Environmental, Health and Safety Studies of Nanoparticles (nano-EHS). J. Nanopart Res 13:1373-1387 